THE ASTROPHYSICAL JOURNAL, 403: L33-L37, 1993 January 20

(C) 1993. The American Astronomical Society. All rights reserved. Printed in U.S.A.

\title{
DISCOVERY OF THE ORBIT OF THE X-RAY PULSAR OAO 1657-415
}

Deepto Chakrabarty, John M. Grunsfeld, ${ }^{1}$ Thomas A. Prince, and Lars Bildsten

Division of Physics, Mathematics, and Astronomy, California Institute of Technology, Pasadena, CA 91125

Mark H. Finger, ${ }^{2}$ Robert B. Wilson, Gerald J. Fishman, and Charles A. Meegan

Space Science Laboratory, NASA/Marshall Space Flight Center, Huntsville, AL 35812

AND

William S. Paciesas

Department of Physics, University of Alabama, Huntsville, AL 35809

Received 1992 September 28; accepted 1992 November 5

\section{ABSTRACT}

Timing observations of the $38 \mathrm{~s}$ accreting X-ray pulsar OAO 1657-415 made with the BATSE large-area detectors on the Compton Gamma Ray Observatory have revealed a binary orbit with an X-ray eclipse by the stellar companion. Arrival time analysis of $20-60 \mathrm{keV}$ data yielded the following best-fit orbital elements: $P_{\text {orb }}=10.4436 \pm 0.0038, a_{x} \sin i=106.0 \pm 0.5 \mathrm{lt}-\mathrm{sec}, e=0.104 \pm 0.005, \omega=93^{\circ} \pm 5^{\circ}, T_{\pi / 2}=\mathrm{JD} 2,448,516.49$ \pm 0.05 TDB. From the pulsar mass function $f_{x}(M)=11.7 \pm 0.2 M_{\odot}$ and the measured eclipse half-angle $\theta_{e}=$ $29.7 \pm 1.3$, we infer that the stellar companion is a supergiant of spectral class B0-B6. If the companion can be identified and its orbital velocity measured, the neutron star mass can be constrained. Both intrinsic spin-up $\left(\dot{P} / P \approx-8 \times 10^{-3} \mathrm{yr}^{-1}\right)$ and spin-down $\left(\dot{P} / P \approx 2 \times 10^{-3} \mathrm{yr}^{-1}\right)$ of the pulsar were measured during our observation.

Subject headings: binaries: close — binaries: eclipsing - pulsars: individual: OAO 1657-415 stars: neutron - X-rays: stars

\section{INTRODUCTION}

There are currently six known eclipsing X-ray binary pulsars with well-determined orbits and optical spectroscopy, all of which yield important constraints on the neutron star mass range (see Nagase 1989). These measurements are complementary to the more accurate ones made in binary radio pulsar systems and might point out any evolutionary differences between these two types of systems. This Letter reports the detection of orbital motion and X-ray eclipses for a seventh $\mathrm{X}$-ray binary pulsar, OAO $1657-415$. Optical identification of the undiscovered companion followed by orbital phase spectroscopy and photometry will provide constraints on the neutron star mass.

The X-ray source OAO $1657-415\left(l=344^{\circ}, b=0.3\right)$ was first detected by the Copernicus satellite (Polidan et al. 1978) in the 4-9 keV range and was initially misidentified as the companion to the massive spectroscopic binary V861 Sco. Subsequent observations by the $H E A O I$ satellite (Byrne et al. 1979; Armstrong et al. 1980) and the Einstein Observatory (Parmar et al. 1980) yielded a position error box which excludes V861 Sco. HEAO 1 observations also showed $38.22 \mathrm{~s}$ pulsations in the 1-40 keV (White \& Pravdo 1979) and 40-80 $\mathrm{keV}$ (Byrne et al. 1981) X-ray flux. More recent observations have shown both spin-up and spin-down of the pulse period (Kamata et al. 1990; Gilfanov et al. 1991; Mereghetti et al. 1991; Sunyaev et al. 1991).

We have made daily pulse period measurements of OAO 1657-415 with the Burst and Transient Source Experiment (BATSE) on the Compton Gamma Ray Observatory (GRO) since 1991 April as part of a joint MSFC/Caltech pulsar detec-

\footnotetext{
${ }^{1}$ Current address: NASA/Johnson Space Center, Code CB, Houston, TX 77058.

${ }^{2}$ Astronomy Programs, Computer Sciences Corporation.
}

tion program. These observations show clear evidence of binary motion and eclipses of the X-ray source by its companion. Preliminary results of a pulse period analysis were given by Finger et al. (1992). In this Letter, we report on an accurate determination of the orbital parameters using an arrival time analysis. The implications for the stellar type of the stillunidentified companion are outlined.

\section{OBSERVATIONS AND TIMING ANALYSIS}

BATSE consists of eight identical uncollimated detector modules arranged on the corners of the GRO spacecraft, providing an all-sky monitor of hard $\mathrm{X}$-ray and $\gamma$-ray flux (Fishman et al. 1989). Except for source occultation by Earth, the instrument provides nearly continuous observations of discrete sources. We are reporting on observations with the BATSE large-area detectors (LADs), each of which contains a $\mathrm{NaI}(\mathrm{Tl})$ scintillation crystal $1.27 \mathrm{~cm}$ thick and $50.8 \mathrm{~cm}$ in diameter, viewed in a light collection housing by three $12.7 \mathrm{~cm}$ diameter photomultiplier tubes. The LADs have an effective energy range of $20 \mathrm{keV}-1.8 \mathrm{MeV}$. In our analysis, only the data in the approximate range $20-60 \mathrm{keV}$ were used, where each LAD has an effective area of $\approx 1000 \mathrm{~cm}^{2}$.

A number of standard data types are generated from the LADs at various time and energy resolutions. The DISCLA data contain the photon count rate from the discriminators of each LAD in four energy channels at $1.024 \mathrm{~s}$ resolution. Our analysis of OAO $1657-415$ is based on the lowest DISCLA energy channel data during the period 1991 April 23-1992 March 3 (MJD $\left.{ }^{3} 48370-48685\right)$. DISCLA data are available for this entire period except for short segments during South Atlantic Anomaly crossings when the high voltage to the detectors was turned off, and for occasional data gaps due to telemetry and onboard tape recorder errors.

${ }^{3}$ Modified Julian Date $=$ JD $-2,400,000.5$. 
The DISCLA data were processed to construct, for each day, a single time series at $1.024 \mathrm{~s}$ resolution on which a timing analysis was performed. For any given GRO orientation OAO $1657-415$ is visible in four detectors except when occulted by Earth (once each orbit). Only data from those detectors were analyzed, using intervals when the source was not occulted. A model of the background in each of the four detectors was generated by passing the data through a low-pass filter; this was subtracted from the detector signals to remove the large (factor of 2) variations in count rate due to the changing background in the $\approx 93$ minute orbit of $G R O$ around Earth. The signals from the four detectors were then optimally weighted for the view direction of OAO $1657-415$ and combined to form a single time series. The spacecraft times of the count rate bins were transformed to arrival times at the solar system barycenter using corrections based on the Jet Propulsion Laboratory DE200 solar system ephemeris (see Taylor \& Weisberg 1989).

Preliminary timing analysis was performed by producing a Fourier power spectrum for each 1 day time series and searching a small period range near the previously observed pulse period of OAO $1657-415$. Since the LADs are uncollimated, and other bright X-ray sources are in the large field of view, this analysis used only the pulsed flux information contained in the data. Having optimized the time series for the view direction of OAO $1657-415$ and searched a narrow range of periods near $38 \mathrm{~s}$, we attributed any statistically significant signal to OAO $1657-415$. A strong signal was detected on approximately $\frac{2}{3}$ of the days, and the resulting period history was used to derive a preliminary orbit.

Using the preliminary orbit as a provisional ephemeris, a pulse profile template was constructed by folding data from 71 days when the signal was strong. We selected these days from an interval (MJD 48370-48460) where the source showed roughly linear spin-up. We then folded 0.2 day segments of our time series at the pulsar ephemeris period and cross-correlated with the template to obtain pulse times of arrival (TOAs) for each segment. These TOAs were fitted over short intervals using a Taylor expansion for intrinsic changes in the pulse period and a standard model for the orbital Doppler delays. Writing the pulse emission times as (e.g., Deeter, Boynton, \& Pravdo 1981)

$$
t_{n}^{\prime}=t_{0}^{\prime}+n P_{0}+\frac{1}{2} n^{2} \dot{P} P_{0},
$$

where $t_{0}^{\prime}$ is the emission time epoch and $P_{0}$ and $\dot{P}$ are the pulse period and its derivative at $t_{0}^{\prime}$, the arrival times can be represented by

$$
t_{n}=t_{n}^{\prime}+f\left(t_{n}^{\prime}\right),
$$

where $f\left(t_{n}^{\prime}\right)$, the orbital Doppler delay, is parametrized by five Keplerian orbital parameters: projected semimajor axis $a_{x} \sin i$ (where $i$ is the inclination angle between the line of sight and the orbital angular momentum vector, defined to lie in the first quadrant), orbital period $P_{\text {orb }}$, eccentricity $e$, longitude of periastron $\omega$, and orbital epoch ${ }^{4} T_{\pi / 2}$ (defined as the epoch where the mean longitude is $90^{\circ}$ ).

Determination of the neutron star orbital elements from the observed pulse history is complicated by the need to decouple the effects of the orbital Doppler delays and intrinsic changes in the neutron star rotation rate. Preliminary fits indicated that

\footnotetext{
${ }^{4}$ See Deeter et al. (1981) for a discussion of the advantages of this particular choice of orbital epoch.
}

$\dot{P}$ changed over orbital time scales. Thus, we have employed a " hybrid" approach to determining the orbit. The gaps in our data caused by eclipses of the X-ray source over its 10.4 day orbit naturally divide our TOA data into $\sim 8$ day segments separated by $\sim 2$ days. The five orbital parameters were fitted simultaneously for all the segments, along with an independent $t_{0}^{\prime}, P_{0}$, and $\dot{P}$ for each segment. This approach is especially attractive because it does not require an unambiguous pulse count to be maintained across eclipse intervals. To minimize the effect of rotation fluctuations on the orbital parameters, we confined our analysis to an interval where the pulse period history could be approximately represented by a single $\dot{P}$ term (MJD 48370-48460).

\section{RESULTS}

Table 1 presents our best-fit orbital elements, measured eclipse information, and several derived quantities. Figure 1 shows the best-fit Doppler delay curve for OAO 1657-415 with our best model of the intrinsic spin period variations removed. The fit residuals are plotted on a $5 \times$ expanded scale. Figure 2 shows the spin period history of OAO $1657-415$ for days MJD 48370-48685, where our best-fit orbital Doppler delays have been removed. OAO $1657-415$ exhibited both spin-up at $\dot{P} / P \approx-8 \times 10^{-3} \mathrm{yr}^{-1}$ and spin-down at $\dot{P} / P \approx$ $2 \times 10^{-3} \mathrm{yr}^{-1}$. The long-term trend since 1979 has been for secular spin-up with $\dot{P} / P \approx-10^{-3} \mathrm{yr}^{-1}$.

A Monte Carlo analysis was used to estimate the singleparameter $1 \sigma$ confidence limits for the orbital elements by generating simulated data sets with $\sigma_{\text {sim }}=2 \mathrm{~s}$ Gaussian errors in the TOAs. We chose $\sigma_{\text {sim }}$ to be larger than the RMS arrival time fit residual $\sigma_{\mathrm{rms}}=1.5 \mathrm{~s}$ and the typical TOA measurement uncertainty $\sigma_{\text {toa }} \sim 1 \mathrm{~s}$ in order to allow for the possibility that red noise fluctuations in the pulsar rotation may have contributed to the parameter uncertainties. We believe this ad hoc approach correctly bounds the parameter uncertainties even though an explicit red noise model was not included in our simulations. A more detailed study of red noise fluctuations in the rotation of OAO $1657-415$ will be presented elsewhere. Boynton et al. (1986) have modeled such variability in Vela X-1 as a random walk (red noise) in spin frequency due to white

TABLE 1

\begin{tabular}{|c|c|c|}
\hline Parameter & Symbol & Value $^{\mathbf{a}}$ \\
\hline $\begin{array}{l}\text { Best-fit orbital parameters and } \\
\text { eclipse measurements: } \\
\text { Orbital period } \ldots \ldots \ldots \ldots \ldots \\
\text { Projected semimajor axis } \ldots \ldots \\
\text { Eccentricity } \ldots \ldots \ldots \ldots \ldots \ldots \ldots \\
\text { Longitude of periastron } \ldots \ldots \ldots \\
\text { Orbital epoch } \ldots \ldots \ldots \ldots \ldots \ldots \\
\text { Eclipse ingress }{ }^{\mathrm{b}} \ldots \ldots \ldots \ldots \ldots \ldots \\
\text { Eclipse egress }^{\mathrm{b}} \ldots \ldots \ldots \ldots \ldots \ldots\end{array}$ & $\begin{array}{l}P_{\text {orb }} \\
a_{x} \sin i \\
e \\
\omega \\
T_{\pi / 2} \\
l_{e, \text { in }} \\
l_{e, \text { out }}\end{array}$ & $\begin{aligned} 10.4436 & \pm 0.0038 \\
106.0 & \pm 0.5 \mathrm{lt}-\mathrm{sec} \\
0.104 & \pm 0.005 \\
93^{\circ} & \pm 5^{\circ} \\
\text { JD } 2,448,516.49 & \pm 0.05 \text { TDB } \\
57.1 & \pm 1.8 \\
116^{\circ} .5 & \pm 1.8\end{aligned}$ \\
\hline $\begin{array}{l}\text { Derived quantities: } \\
\text { Pulsar mass function } \ldots \ldots \ldots \ldots \\
\text { Eclipse half-angle } \ldots \ldots \ldots \ldots \ldots\end{array}$ & $\begin{array}{l}f_{x}(M) \\
\theta_{e}\end{array}$ & $\begin{array}{l}11.7 \pm 0.2 M_{\odot} \\
29.7 \pm 193\end{array}$ \\
\hline $\begin{array}{l}\text { Inferred constraints }: \mathrm{c} \\
\text { Orbital inclination } \ldots \ldots \ldots \ldots \\
\text { Companion mass } \ldots \ldots \ldots \ldots \ldots \\
\text { Companion radius } \ldots \ldots \ldots \ldots \ldots \\
R_{c} \text {-Roche radius ratio } \ldots \ldots \ldots \ldots\end{array}$ & $\begin{array}{l}i \\
M_{c} \\
R_{c} \\
R_{c} / R_{L}\end{array}$ & $\begin{array}{c}\gtrsim 60^{\circ} \\
14-18 M_{\odot} \\
25-32 R_{\odot} \\
\quad \gtrsim 0.85\end{array}$ \\
\hline
\end{tabular}

OAO 1657-415 PARAMETERS

a Quoted uncertainties are single-parameter $1 \sigma$ confidence limits.

b Mean longitude.

${ }^{c}$ For $M_{x}=1.4 M_{\odot}$ and the companion inside its critical lobe at periastron. 


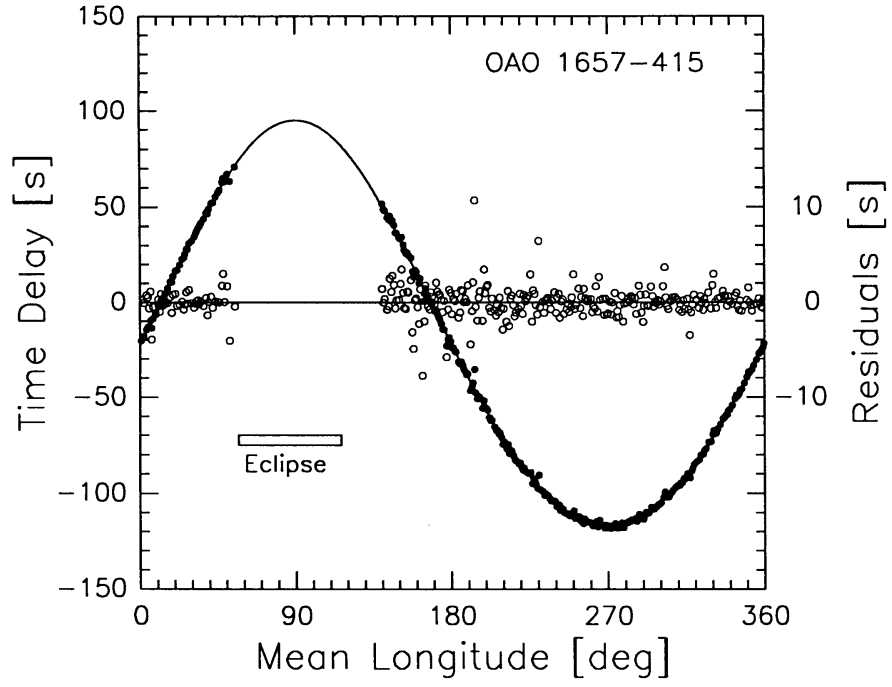

Fig. 1.-Doppler delays for the pulse arrival times as a function of the 10 d4 436 orbit of OAO $1657-415$ after removing our best model for the intrinsic variations in spin period. The data plotted are from the interval MJD 48370-48460. The solid circles are the delay times, the solid curve is the best-fit orbital model, and the open circles are the residuals $(5 \times$ expanded scale).

noise torque fluctuations and found that torque noise was the dominant contribution to the orbital parameter uncertainties.

An eclipse centered near periastron $\left(\omega=93^{\circ} \pm 5^{\circ}\right)$ is evident in Figure 1. We have measured the eclipse duration by dividing our entire data set into 100 orbital phase bins and folding each phase bin at the pulsar spin period. Each of these light curves was correlated with our template to determine in which orbital phase bins no signal was seen. To obtain further precision, we then repeated the process, shifting the orbital phase "zero" by one-half phase bin. We measured eclipse ingress at mean longitude $l_{e, \text { in }}=57^{\circ} .1 \pm 1.8$ and eclipse egress at $l_{e, \text { out }}=116^{\circ} .5$ \pm 1.8 , yielding an eclipse half-angle $\theta_{e}=29.7 \pm 1.3$. A comparison of the BATSE-derived eclipse ephemeris with the orbital phase (assuming our values of $P_{\text {orb }}$ and $T_{\pi / 2}$ ) for the 10 previous observations ${ }^{5}$ of pulsed flux from OAO $1657-415$

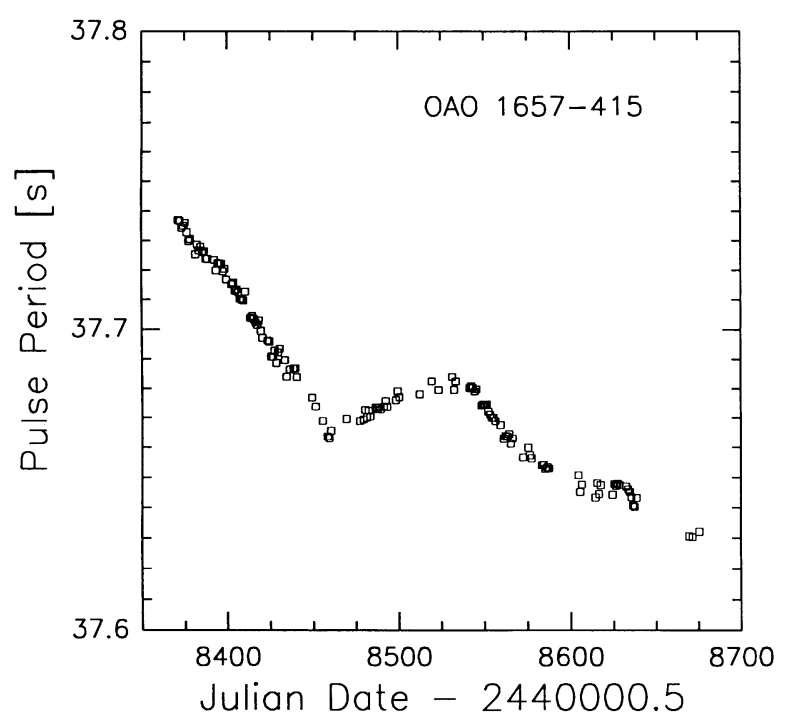

Fig. 2.-Spin period history for OAO 1657-415 derived from BATSE data with the orbital Doppler shifts removed. Only data for which a significant signal was detected are shown.
(White \& Pravdo 1979; Parmar et al. 1980; Kamata et al. 1990; Mereghetti et al. 1991; Gilfanov et al. 1991; Sunyaev et al. 1991) shows that none of these detections lies inside a predicted eclipse interval, providing an additional consistency check on our orbit fit. In particular, the 1983 July Tenma detections of pulsed flux (Kamata et al. 1990) restrict the allowed values of orbital period consistent with our data to the interval $10.4420 \lesssim P_{\text {orb }} \lesssim 10$ d 4450 . The previous observations provide a weak limit on the orbital evolution rate, $\left|\dot{P}_{\text {orb }} / P_{\text {orb }}\right| \lesssim 10^{-5} \mathrm{yr}^{-1}$.

We can use the derived orbital parameters to constrain the undiscovered companion's mass and radius. For a particular choice of neutron star mass $M_{x}$, the X-ray mass function

$$
f_{x}(M) \equiv \frac{4 \pi^{2}\left(a_{x} \sin i\right)^{3}}{G P_{\mathrm{orb}}^{2}}=\frac{\left(M_{c} \sin i\right)^{3}}{\left(M_{x}+M_{c}\right)^{2}}=11.7 \pm 0.2 M_{\odot}
$$

(derived from our measured values of $a_{x} \sin i$ and $P_{\text {orb }}$ ) yields a lower limit for the companion mass $M_{c}$, for which $\sin i=1$. Choosing a value for $M_{c}$, we calculate $\sin i$ and the mean orbital separation $a=a_{x}+a_{c}$. We can then use the measured eclipse angle to estimate the radius of the companion. For an eclipse centered at periastron of an eccentric orbit, we have

$$
R_{c} \approx \frac{a\left(1-e^{2}\right)}{1+e \cos \phi_{e}}\left(\cos ^{2} i+\sin ^{2} i \sin ^{2} \phi_{e}\right)^{1 / 2},
$$

where $\phi_{e}$, the eclipse half-angle in true longitude, can be written in terms of the usual eclipse half-angle $\theta_{e}$ using the standard elliptic expansion for true anomaly in terms of mean anomaly $^{6}$ (Brouwer \& Clemence 1961),

$$
\phi_{e}=\theta_{e}+2 e \sin \theta_{e}+\frac{5}{4} e^{2} \sin 2 \theta_{e}+O\left(e^{3}\right) .
$$

By requiring that $R_{c}$ be less than the radius of the critical potential lobe $R_{L}$ at periastron, we obtain an upper limit on the companion mass. To show the largest possible parameter space, we only consider slow rotation of the companion $\left(P_{c} \gg\right.$ $\left.P_{\text {orb }}\right)$, in which case the Joss \& Rappaport (1984) parameterization of $R_{L}$ simply scales to the separation at periastron (Avni 1976).

Figure 3 shows the resulting $M_{c}-R_{c}$ curves under this constraint and the assumption that $M_{x}>1.0 M_{\odot}$. The dark solid lines bound the allowed region while the light solid lines indicate the $M_{c}-R_{c}$ relations for different neutron star masses with varying inclinations. The allowed region for corotating (i.e., $P_{c}=P_{\text {orb }}$ ) companions is extremely small. The dark solid line on the left corresponds to inclination $i=90^{\circ}$, while the dark solid curve at the top is the boundary for $R_{c}<R_{L}$. The dark solid curve at the bottom corresponds to $M_{x}=1.0 M_{\odot}$. If we assume a neutron star mass of $M_{x}=1.4 M_{\odot}$, then a nonrotating companion has $14 M_{\odot} \lesssim M_{c} \lesssim 18 M_{\odot}$ and $25 R_{\odot} \lesssim$ $R_{c} \lesssim 32 R_{\odot}$. These estimates are based on the smallest eclipse angle consistent with our measurements $\left(\theta_{e} \approx 28^{\circ}\right)$, which places the weakest limits. If the star is inside of the critical lobe at periastron, then our measurements also constrain $i \gtrsim 60^{\circ}$, and $R_{c} / R_{L} \gtrsim 0.85$. Since the question of overflow at periastron is a dynamical one when the star is close to filling the critical lobe, we have arbitrarily extended the $M_{c}-R_{c}$ relations to the point where $R_{c}=R_{L}$ at $0.95 a$ (dashed lines). This provides an

\footnotetext{
${ }^{5}$ We included a 1983 detection by the EXOSAT ME experiment using data from the High Energy Astrophysics Science Archive Research Center at NASA/Goddard Space Flight Center.

${ }^{6}$ For an eclipse centered at periastron, $\theta_{e}$ and $\phi_{e}$ are identical to the eclipse egress value of the mean and true anomalies, respectively.
} 


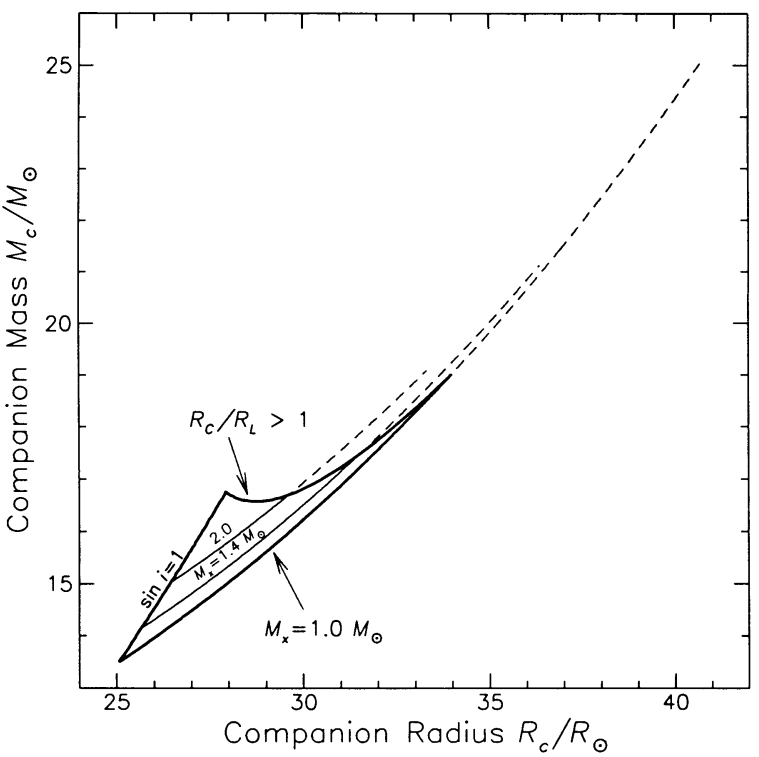

FIG. 3.-Constraints on the mass and radius of the companion of OAO $1657-415$, based on the measured orbital parameters and assuming an eclipse half-angle $\theta_{e}=28^{\circ}$. The region inside of the dark solid curves shows the allowed $M_{c}-R_{c}$ values for inclination $i<90^{\circ}$ (left curve), for a companion which does not overfill its critical lobe at periastron (top curve), and for neutron star mass $M_{x}>1 M_{\odot}$ (bottom curve). The light solid lines indicate the $M_{c}-R_{c}$ relation for neutron star mass $M_{x}=1.4$ and $2.0 M_{\odot}$. The dashed lines show the extended $M_{c}-R_{c}$ relations if the star is allowed to overflow the critical lobe at periastron.

extreme upper limit since it implies that $R_{c}>R_{L}$ for $\sim 40 \%$ of the orbit.

The constraints on $M_{c}$ and $R_{c}$ allow us to locate the star on the theoretical H-R diagram (see Maeder \& Meynet 1989), yielding a blue supergiant of stellar type B0-B6 with absolute visual magnitude $M_{v} \approx-6.3$, corresponding to luminosity class Ia-Iab.

\section{DISCUSSION}

We have measured the eccentric binary orbit and eclipse duration of the accretion-powered X-ray pulsar OAO 1657-415. Our measurements imply that the mass-giving companion is a blue supergiant of mass $14-18 M_{\odot}$. Optical identification of the companion followed by a measurement of its orbital velocity and accurate orbital phase photometry would place important constraints on the neutron star mass.

The distance to OAO $1657-415$ is still unknown. However, we can set a lower limit to the distance from our observation of steady spin-up. Accretion is difficult, if not impossible, when the Alfvén radius (where the kinetic energy density of the accreting matter is equal to the magnetic energy density) lies outside the corotation radius $r_{\text {co }}$ (where the spin period is equal to the Keplerian period). The maximum torque exerted on the neutron star, $N_{\max }=\dot{M}\left(G M_{x} r_{\text {co }}\right)^{1 / 2}$, occurs when the Alfvén radius and the corotation radius are equal. The observed values of the pulsar spin period and its derivative during the steady spin-up interval (MJD 48370-48460) then imply

$$
L_{x} \approx \frac{G M_{x} \dot{M}}{R_{x}} \gtrsim 1.6 \times 10^{37} \operatorname{ergs~s}^{-1}\left(\frac{M_{x}}{1.4 M_{\odot}}\right)^{4 / 3}\left(\frac{R_{x}}{10 \mathrm{~km}}\right),
$$

where we have used $I_{x}=(2 / 5) M_{x} R_{x}^{2}$. A preliminary analysis of the 16 channel CONT data from BATSE for the spin-up interval indicates that the spectral index and hard X-ray pulsed flux were similar to the values measured during the 1978 September observation by HEAO 1 (Byrne et al. 1981). Assuming the same pulsed fraction, we can use the total X-ray flux measured during the $H E A O 1$ pointing, $F_{x} \approx 10^{-9} \operatorname{ergs~s}^{-1} \mathrm{~cm}^{-2}$ (White \& Pravdo 1979), to obtain the limit

$$
\begin{aligned}
d \gtrsim 11 \mathrm{kpc}\left(\frac{M_{x}}{1.4 M_{\odot}}\right)^{2 / 3}( & \left.\frac{R_{x}}{10 \mathrm{~km}}\right)^{1 / 2} \\
& \times\left(\frac{F_{x}}{10^{-9} \mathrm{ergs} \mathrm{s}^{-1} \mathrm{~cm}^{-2}}\right)^{-1 / 2},
\end{aligned}
$$

consistent with the source's low galactic latitude.

Corbet (1986) showed that massive X-ray binaries fall into three separate groups when the pulse period is plotted versus the orbital period. Those systems with unevolved Be companions have a strong correlation between the orbital and spin periods (see Waters \& van Kerkwijk 1989 for an updated discussion), while systems with $\mathrm{OB}$ supergiant companions fall into two separate broad regions of the diagram. Systems with optical photometric evidence for accretion disks (LMC X-4, Cen X-3, and SMC X-1; see van Paradijs 1991) and nearly steady spin-up have the shortest spin and orbital periods, while those without such evidence have much longer spin periods $(\gtrsim 100 \mathrm{~s})$ and show torque reversals characteristic of accretion from the wind of the supergiant (Blondin et al. 1990). Interestingly, OAO $1657-415$ falls between these two groups, both on the Corbet diagram and in terms of its observed spin period history. Its short spin period relative to the wind accretors probably results from the long intervals $\left(\sim 100^{d}\right)$ of steady spin-up which we observe. Whether these spin-up episodes are due to transient disk accretion can be resolved through optical photometry, which is sensitive to emission from an accretion disk.

A detailed study of spin period history (including torque fluctuations), flux history, variations of the pulse profile with energy and luminosity, and pulse phase spectroscopy of the $\gamma$-ray emission is in progress.

We would like to acknowledge the significant contributions to this work made by the entire BATSE team at NASA/ Marshall Space Flight Center, and useful discussions with Stuart Anderson and Paul Ray at Caltech. An anonymous referee made several helpful suggestions. This work is funded in part by NASA grants NAGW-1919, NAGW-2920, and NAG 5-1458, and a Lee A. DuBridge fellowship to L. B. funded by the Weingart Foundation.

\section{REFERENCES}

Amstrong, J. T., Johnston, M. D., Bradt, H. V., Cowley, A. P., Doxsey, R. E., Griffiths, R. E., Hesser, J. E., \& Schwartz, D. A. 1980, ApJ, 236, L131

Avni, Y. 1976, ApJ, 209, 574

Blondin, J. M., Kallman, T. R., Fryxell, B. A., \& Taam, R. E. 1990, ApJ, 356, 591

Boynton, P. E., Deeter, J. E., Lamb, F. K., \& Zylstra, G. 1986, ApJ, 307, 545
Brouwer, D., \& Clemence, G. M. 1961, Methods of Celestial Mechanics (New York: Academic)

Byrne, P., et al. 1979, IAU Circ., No. 3368

Byrne, P. F., et al. 1981, ApJ, 246, 951

Corbet, R. H. D. 1986, MNRAS, 220, 1047

Deeter, J. E., Boynton, P. E., \& Pravdo, S. H. 1981, ApJ, 247, 1003 
Finger, M. H., Chakrabarty, D., Grunsfeld, J. M., Prince, T. A., Wilson, R. B., Fishman, G. J., Meegan, C. A., \& Paciesas, W. S. 1992, IAU Circ., No. 5430

Fishman, G. J., et al. 1989, in Proc. GRO Science Workshop, ed. W. N. Johnson (Greenbelt: NASA/GSFC), 2-39

Gilfanov, M., Sunyaev, R., Churazov, E., Babalyan, G., Pavlinskii, M., Yamburenko, N., \& Khavenson, N. 1991, Soviet Astron. Lett., 17, 46

Joss, P. C., \& Rappaport, S. A. 1984, ARA\&A, 22, 537

Kamata, Y., Koyama, K., Tawara, Y., Makishima, K., Ohashi, T., Kawai, N., \& Hatsukade, I. 1990, PASJ, 42, 785

Maeder, A., \& Meynet, G. 1989, A\&A, 210, 155

Mereghetti, S., et al. 1991, ApJ, 366, L23
Nagase, F. 1989, PASJ, 41,

Parmar, A. N., et al. 1980, MNRAS, 193, 49P

Polidan, R. S., Pollard, G. S. G., Sanford, P. W., \& Locke, M. C. 1978, Nature, 275,296

Sunyaev, R., Gilfanov, M., Goldurm, A., \& Schmitz-Frayesse, M. C. 1991, IAU Circ., No. 5342

Taylor, J. H., \& Weisberg, J. M. 1989, ApJ, 345, 434

van Paradijs, J. 1991, in Neutron Stars: Theory and Observation, ed. J. Ventura \& D. Pines (Dordrecht: Kluwer), 289

Waters, L. B. F. M., \& van Kerkwijk, M. H. 1989, A\&A, 223, 196

White, N. E., \& Pravdo, S. H. 1979, ApJ, 233, L121 